\title{
Interview with Irving W Wainer
}
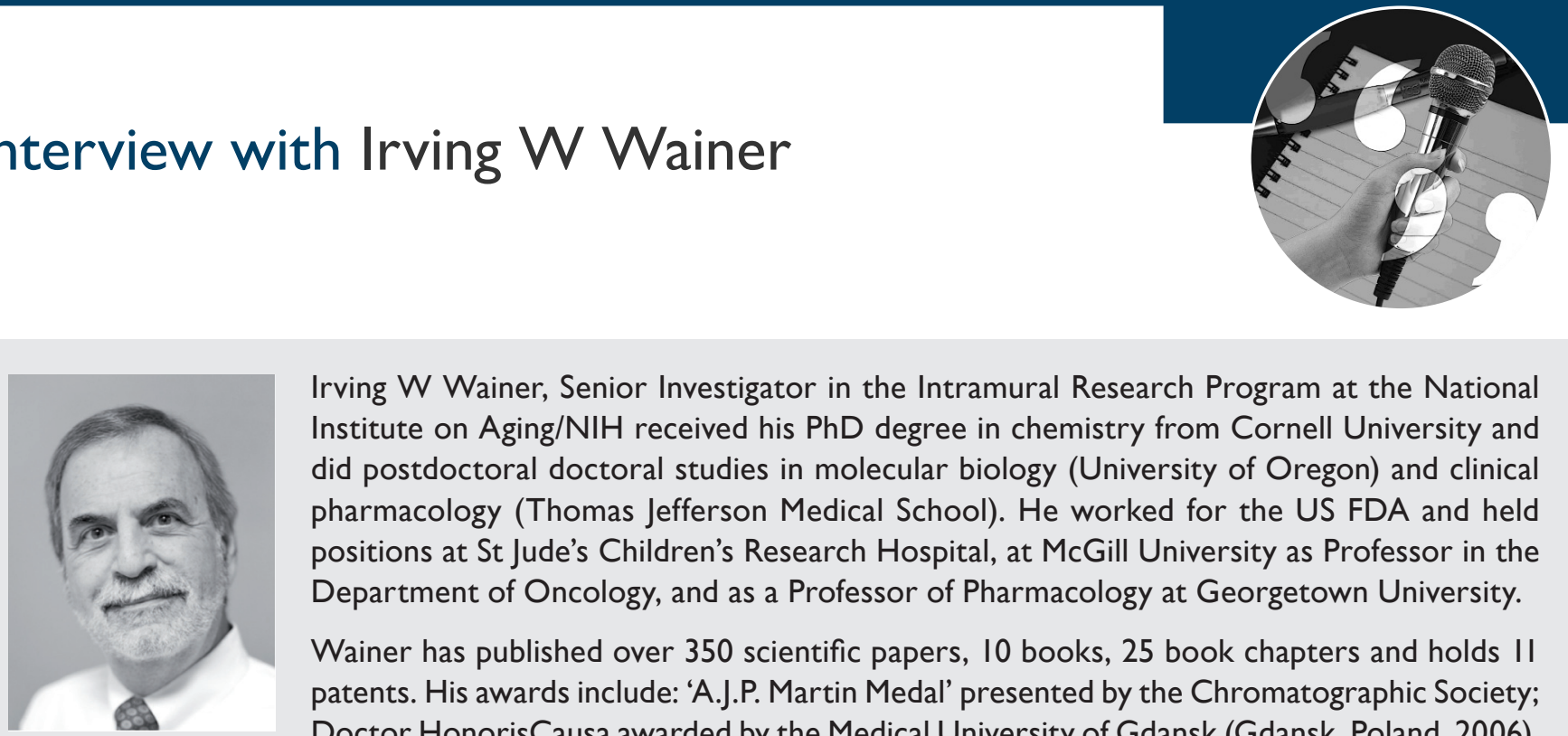

Irving W Wainer, Senior Investigator in the Intramural Research Program at the National Institute on Aging/NIH received his $\mathrm{PhD}$ degree in chemistry from Cornell University and did postdoctoral doctoral studies in molecular biology (University of Oregon) and clinical pharmacology (Thomas Jefferson Medical School). He worked for the US FDA and held positions at St Jude's Children's Research Hospital, at McGill University as Professor in the Department of Oncology, and as a Professor of Pharmacology at Georgetown University.

Wainer has published over 350 scientific papers, 10 books, 25 book chapters and holds II patents. His awards include: 'A.J.P. Martin Medal' presented by the Chromatographic Society; Doctor HonorisCausa awarded by the Medical University of Gdansk (Gdansk, Poland, 2006), Doctor HonorisCausa awarded by the Department of Medicine, University of Liege (Liege, Belgium, 2012), and the 2013 Eastern Analytical Symposium Award for Outstanding Contributions to the Fields of Analytical Chemistry.

Wainer's research includes the development of new therapeutic agents for the treatment of congestive heart failure, cancer, pain and depression, many of which are in the later stages of drug development. His laboratory has also continued the development of cellular membrane affinity chromatography technology, and recent work includes the development of columns containing immobilized forms of the breast cancer resistance protein found in cellular and nuclear membranes and mitochondrial membrane columns. Wainer's laboratory has also continued its study of the effect of disease progression and aging on drug metabolism in critically ill and terminal patients.

Interview was conducted by Lisa Parks, Assistant Commissioning Editor of Bioanalysis.

How did it feel to be presented with the 'Chromatography Forum of the Delaware Valley Dal Nogare Award' at Pittcon 2013?

It was quite an honor to receive the Dal Nogare Award and to be included with the brilliant and accomplished people who have previously received this award. I have followed the work of the Chromatography Forum of the Delaware Valley (CFDV) for many years and had the opportunity to attend a number of the events and courses that they have sponsored. It means a lot to me to have our work recognized by the CFDV. The body of work that has been cited by the CFDV could not have been accomplished without the collaboration of my students, fellows, colleagues and support staff. The people that I have been fortunate enough to work with have made me look good and so I see this award as recognition of a team effort over quite a period of time. It was also a lot of fun and my colleagues made work enjoyable. One additional point, I was the chairman of the HPLC 2002 meeting held in Montreal, Quebec, Canada. This was a very difficult period for international meetings, but we were very successful due, in part, to the support of the CFDV. I would like to take this opportunity to once again thank the CFDV for their support of the HPLC 2002 organizing committee.

Please describe some of the work involved that led to you winning the award at Pittcon 2013.

The work that I have done over my career has centered on stereochemistry and enantioselective separations. We started using enantioselective chromatography during my tenure at the US FDA when the goal was to separate chiral drugs. We did a great deal of work with all of the commercially available HPLC chiral stationary phases (CSP), which led to the publication of ' $A$ Practical Guide to the Selection and Use of HPLC Chiral Stationary Phases' in 1988. The publication of the 'Practical Guide' reflected the beginning of a change in our basic approach to the use of CSP as we started to use chiral separations as a probe of molecular interactions between small molecules and proteins. This approach led to the

\section{Irving W Wainer}

Bioanalytical Chemistry \& Drug Discovery Section, Laboratory of Clinical Investigation, National Institute on Aging, NIH, 25I Bayview Blvd, Baltimore 08B।33, MD 21224, USA

E-mail: wainerir@grc.nia.nih.gov 
development of a series of columns based upon immobilized proteins, drug transporters, receptors and cellular membranes, and the utilization of what we call cellular membrane affinity chromatography (CMAC). The objective of this technique is to study pharmacology and biology within an LC column and to mimic what is happening in the circulatory system. Stereochemistry is a wonderful probe because enantiomers have essentially the same physical and chemical properties, which can be used as a control for the nonspecific interactions occurring during the chromatographic process. For example, if you chromatograph both enantiomers of a chiral drug on a LC column containing a specific protein target and you achieve an enantioselective separation, the observed enantioselectivity is a reflection of the specific and stereoselective interactions between the drug and the protein.

Our work in the chiral separation of drugs merged with another part of my life, basic and clinical pharmacology. We applied HPLC CSP to the development of bioassays in plasma and urine for PK and metabolic studies and to study cellular interactions. This work led to my co-editing of the book "Drug Stereochemistry; Analytical Methods and Pharmacology" in 1988, the third edition was published this year. In 1988, I was also the Founding Editor of the journal Chirality.

Q At what stage in your career did you first become involved in bioanalysis \& what first attracted your attention to this area?

I received my PhD degree from Cornell University where I trained as a synthetic organic chemist with Jerrold Meinwald. As part of my doctoral work, I studied physical and biological chemistry and used these approaches to chemically and biologically characterize the compounds I had synthesized. As a synthetic chemist I had to learn flash chromatography, thin-layer chromatography and GC, so I had a background in analytical methods.

I left graduate school as a synthetic chemist and did postdoctoral studies in molecular biology at the University of Oregon followed by a second fellowship in clinical pharmacology at Thomas Jefferson University in Philadelphia. I worked with John Stambaugh, a clinical oncologist, who was looking for someone to assay plasma drug levels as part of his clinical trials. The arrangement was that I would develop and carryout the necessary bioassays and he would teach me clinical pharmacology, which sounded like a pretty good deal. So, I became a bioanalytical chemist because I wanted to learn clinical pharmacology.

Bioanalytical chemistry provided an excellent opportunity for me as there are a lot of areas of clinical and basic pharmacology that required a chemist's point of view. One of my mentors, the late Goran Schill from Uppsala University, would always tell me, "listen to the data and let the data tell you the story. Never start a project knowing what this data will tell you because you will miss it." The advantage that we have as bioanalytical chemists is that we measure everything that is there and, as long as we are not prejudice, the data will tell the story. In this manner, I have been extremely impressed by the people developing metabolomics and pharmacometabolomics, as they have really showed us how to make sense out of chaos, which is a real strength of being a bioanalytical chemist.

Please can you update us on some of the research activities in which your group is currently engaged?

We are still engaged in research regarding CMAC columns and we are using them now to begin to answer some basic questions in drug discovery. One of our current areas of study is the resistance of some tumors to drug therapy. One form of this resistance - multiple drug resistance - is based upon the ability of cells to export cytotoxic agents using drug-exporter proteins. We have studied these drug exporters by making a series of CMAC columns using cellular membranes obtained from cancer cells and we were able to relate functional resistance to transporter expression. For example, we constructed CMAC columns using membranes from the LN229 glioblastoma (brain cancer) cell line and demonstrated that the resulting column contained three drug exporters: Pgp, MRP and BCRP. Thus, using a single CMAC column we can pattern the interaction of potential inhibitors at all three transporters in a single chromatographic run. In addition, we have recently demonstrated that two of these transporters, Pgp and BCRP, are also expressed on the nuclear membrane on the LN229 cell and have constructed the first nuclear membrane affinity column (NMAC) to study and characterize the effect of nuclear expression on transporter function.

Second, we are interested in cellular metabolism and energy and are working with a group at Tallinn University, Estonia to construct 
mitochondrial membrane columns and mitochondrial reactor columns. We are going to study mitochondrial function in healthy and diseased states using these columns.

We are also still actively studying clinical pharmacology. For example, we have recently collaborated with Carlos Zarate from the National Institute of Mental Health, NIH, in a study of the use of $(R, S)$-ketamine in the treatment of depression. The results demonstrate that $70 \%$ of patients with bipolar depression responded to low doses of ketamine within $4 \mathrm{~h}$ of drug administration. We analyzed the plasma samples from this study and undertook a complete PK/PD analysis of the data. The results demonstrated that downstream metabolites of $(R, S)$-ketamine play a major role in the observed clinical responses. My laboratory is now working on the pharmacological and clinical characterization of these downstream metabolites and their potential clinical applications in the treatment of depression.

Q During your time at the US FDA, your duties included the development of the FDA's program on the stereoisomeric purity of drugs. What challenges do we face in the separation of stereoisomeric drugs \& how has your research helped to overcome this?

As I stated earlier, my goal at the FDA was to determine the stereoisomeric purity of chiral drugs. The key issue was how to separate the isomers. Our main approach was using HPLC CSP. The publication of my 'Guide' in 1988 demonstrates that we had achieved the primary goal and that by using HPLC CSP we were able to determine stereoisomeric purity, as well as achieve it via preparative separations. This work helped shape the FDA's policy on chiral drugs and I am proud of this contribution to improving the effectiveness of therapeutic agents.

Although the ability to use HPLC CSP to resolve chiral drugs was established by the late 1980 's, this did not mean that the development of HPLC CSP technology stopped. What I think has happened in recent years is that we have got better at doing what we could already do. Now, you can achieve HPLC CSP chiral separations with greater speed and higher efficiency. These are quantitative not qualitative changes in the technology. In my laboratory, chiral separations have gone from a goal to a probe, and the objectives are to study chiral recognition mechanisms and ligand-protein or protein-protein interactions, which was a natural progression for us.
Is there a recently published paper in the bioanalytical field that has caught your attention?

A single paper, no, but I am impressed by the wonderful work being carried out in the area of metabolomics and pharmacometabolomics, which started with the seminal papers of Jeremy Nicholson and Ian Wilson. I am particularly intrigued by the use of pharmacometabolomics data to read back to genomics and to the basic sources of therapeutic response to disease. I am interested in the whole metabolomics field and I cannot really pick out any particular paper as my response to most of the publications is 'boy, I wish I could do that!' It is exciting to look at where we are going as increased chromatographic efficiency and online structural identification enables us to produce patterns that can be associated with drug response or nonresponse, as well as healthy and disease states.

In your opinion, what have been the most important milestones during your time as a research scientist in the bioanalytical field?

The first one is this explosion of HPLC CSP in the early 1980s. When you look at it from a historical perspective this was a science that came out of almost nowhere and was fully matured in less than 10 years reaching into every area of pharmaceutical research. The accepted paradigm became that a racemic mixture was actually a formulation of two independent compounds and that anytime you have a formulation of two compounds you have to be able to prove the efficacy and toxicity of both. So if you came up with a racemic mixture, by the end of the 1980s you could not say to the FDA and other regulatory agencies, "I know it is a racemate but I do not know the efficacy and toxicity of the individual enantiomers of the mixture", as the response from the FDA would be "we know you can separate them, we know it can be done so do it." This made quite a revolutionary change in how we make pharmaceuticals and how we think about making pharmaceuticals. I feel that my work made a significant contribution to this policy.

Second, the increased efficiency of LC separation and the development of hyphenated techniques. Now we are able to analyze biological matrices for parent drugs, metabolites and disease and response markers. Thus, our ability 
as bioanalytical chemists to contribute to our understanding of health and disease and treatment of diseases is unlimited because our ability to measure has increased.

Q In the next 5-10 years, what particular element of bioanalysis do you see advancing the most \& why?

Metabolomic and pharmacometabolomic research as they are clearly linked to our better understanding of how humankind works. It is the next logical progression, reading up from the genome, to the proteome, to the metabolome, to the pharmacometabolome and all the other 'omics that are out there.

The increased selectivity and sensitivity in drug bioanalysis is important as the new therapeutic agents are more potent, presenting increased bioanalytical challenges. One example of this is a new drug we are developing in my laboratory that is active in the treatment of brain cancer at picomolar levels. Modern bioanalytical technology allows us to not only measure the plasma disposition of this drug, but to also follow the concentrations of the agent in the tumor tissues and correlate both measurements to response. This aspect of bioanalysis is going to continue to be more and more challenging and solving these challenges is going to be great fun!

What words of encouragement would you give young scientists who might be envisioning a career in bioanalytical science?

When I look back at my scientific career, the two part phrase that seems best to describe this journey is "whenever someone says "everyone knows that"... stop listening'. When I was told that "everybody knows that you cannot immobilize enzymes on a chromatographic column, keep them active and perform enzyme kinetic studies", I did not listen and developed a whole series of immobilized enzyme reactors for online pharmacological, metabolic and synthetic uses. I was even told that "everyone knows that you cannot separate chiral compounds using CSPs", and I think that it is clear that I did not listen to that advice. I cannot count the number of times that 'experts' have told me what everybody knows that. The best thing to do is to smile politely and then go and do what you want to do. If it fails, then maybe everyone does know that, and that is ok. It is particularly important for young scientists to not let people fence in their ideas and to put boundaries around a new vision of science, society or life. These limits are usually based upon generally accepted explanations and, while I can understand that, I always have this vision of these people running after Columbus as he sails off to the new world shouting 'do not go, you will drop off the edge of the world'. Fortunately, he didn't listen to them!

\section{Disclaimer}

The opinions expressed in this interview are those of the interviewee and do not necessarily reflect the views of Future Science Ltd.

\section{Financial \& competing interests disclosure} The author has no relevant affiliations or financial involvement with any organization or entity with a financial interest in or financial conflict with the subject matter or materials discussed in the manuscript. This includes employment, consultancies, honoraria, stock ownership or options, expert testimony, grants or patents received or pending, or royalties.

No writing assistance was utilized in the production of this manuscript. 